Теорія Ймовір. та Матем. Статист. Вип. 76, 2007
Theor. Probability and Math. Statist.

No. 76, 2008, Pages 141-153

S 0094-9000(08)00738-2

Article electronically published on July 16, 2008

\title{
RANDOM MOTIONS IN INHOMOGENEOUS MEDIA
}

UDC 519.21

\author{
E. ORSINGHER AND N. E. RATANOV
}

\begin{abstract}
Space inhomogeneous random motions of particles on the line and in the plane are considered in the paper. The changes of the movement direction are driven by a Poisson process. The particles are assumed to move according to a finite velocity field that depends on a spatial argument.

The explicit distribution of particles is obtained in the paper for the case of dimension 1 in terms of characteristics of the governing equations. In the case of dimension 2 , the distribution is obtained if a rectifying diffeomorphism exists.
\end{abstract}

\section{INTRODUCTION}

Consider a transport equation of the following form (see, for example, $9,10,21,22$ ):

$$
\frac{\partial p}{\partial t}(x, v, t)+v \cdot \nabla p(x, v, t)=-\mu p(x, v, t)+\mu \int_{V} T\left(v, v^{\prime}\right) p\left(x, v^{\prime}, t\right) d v^{\prime} .
$$

Here $p(x, v, t)$ denotes the density of particles at a spatial position $x \in \mathbb{R}^{n}$ moving with velocity $v \in V \subset \mathbb{R}^{n}$ at time $t \geq 0$ and $\mu$ is a constant turning rate. The turning kernel $T\left(v, v^{\prime}\right)$ is the probability that the velocity jumps from $v^{\prime}$ to $v$ provided the jump occurs.

This general model applies in various fields from thermal conductivity and photon transport in a highly scattering medium [5, 22, to description of the motion of biological organisms [4, 9, 10]. As mentioned in 22], the solution of the full transport equation can only be found numerically. Thus it is natural to seek stochastic models and the corresponding differential equations that are simpler than the general equation (1.1). We want these models to be analytically tractable but still retain significant features of the underlying physics.

In the one-dimensional case, this equation is equivalent to the so-called telegraph equation, which is a widespread research project beginning from the middle of the 19th century (see 5 for a survey of the history of the question). However, the reduction of the process with jump velocities to the telegraph equation is possible in the one-dimensional case only. Even if only four directions are allowed in the plane, the system does not reduce to the telegraph equation in any scaling (see [10, 13]). Nevertheless the equations can be solved in quadratures in the latter case.

An alternative approach to the transport equation (1.1) leads to the telegraph equation for all dimensions (see [22, 16]). These two approaches do not coincide. We explore the first approach in this paper.

It is worthwhile mentioning at this point that almost all known literature on this subject is devoted to equations of the type (1.1) with constant coefficients, while the

2000 Mathematics Subject Classification. Primary 60K99; Secondary 62G30, 35L25, 60C05.

Key words and phrases. Bessel functions, Poisson process, rectifying diffeomorphism, hyperbolic equations, telegraph process. 
medium is highly inhomogeneous in many physical and biological applications (see 2, 4, 22]), and thus the underlying equations contain varying coefficients.

In a recent survey paper, Weiss wrote that "... little is known about more general equations with ... space dependent coefficients" ([22, p. 394]). We try to fill the gap in this paper.

We develop the idea of a rectifying diffeomorphism to construct explicit solutions for inhomogeneous media. The results are illustrated by examples of physical and biological processes. In particular, the diffusions in the medium with circular (see Example 3 in Section 4.3 below) or cellular structure (see 2 or Section 5 below) can be given in terms of velocity-jump processes with varying velocity.

The results presented in this paper supply the mathematical base for developing the theory of travelling waves in reaction-diffusion systems. The existing theory is highly motivated in biology and physics [4, 9, 10, but it is developed mainly for homogeneous media. An extension of the theory to inhomogeneous media is a problem of great importance for applications (see [19, 20]).

\section{RAndom motions governed By Poisson Flows}

Let $(\Omega, \mathfrak{F}, \mathrm{P})$ be a complete probability space. Let $\tau_{1}, \tau_{2}, \ldots$ be random times that form a Poisson flow with rate $\mu>0$. Let $N(t)=\max \left\{i: \tau_{i}<t\right\}, t \geq 0, N(0)=0$, denote the total number of Poisson events occurring up to time $t$. This paper is concerned with various types of motions on the line and in the plane. We assume that abrupt changes of the movement direction occur at the Poisson times $\tau_{i}, i=1,2, \ldots$.

Investigations of this model begin with the famous works by Goldstein [3] and Kac [6. 7]. Further developments are due to both probabilists and physicists (see, for example, [12, 17]). Planar random motions are considered in [8, 14, 15].

The one-dimensional version of this model with constant velocity is called the telegraph process. This process is defined by

$$
U(t)=\xi \int_{0}^{t}(-1)^{N(s)} d s,
$$

where the random variable $\xi$ (taking values \pm 1 with equal probabilities) and the Poisson process $N(\cdot)$ are independent. The stochastic process (2.1) corresponds to the motion of a particle that starts from the origin and moves on the line with unit velocity. The particle changes the direction of movement at times $\tau_{i}, i=1,2, \ldots$. It is clear that $U(t)$ is the position of the moving particle at time $t$.

The distribution of the process $U(t)$ defined by (2.1) is well known and can be written in the following form:

$$
\begin{aligned}
q_{1}(y, t) \equiv & q_{1}^{\mu}(y, t) \\
= & \frac{e^{-\mu t}}{2}[\delta(y-t)+\delta(y+t) \\
& \left.\quad+\left(\mu I_{0}\left(\mu \sqrt{t^{2}-y^{2}}\right)+\frac{\partial}{\partial t} I_{0}\left(\mu \sqrt{t^{2}-y^{2}}\right)\right) \cdot \chi_{[-t, t]}(y)\right]
\end{aligned}
$$

(see, for example, [12]). Here and in what follows the symbol $\chi_{\Delta}(\cdot)$ stands for the indicator of a set $\Delta$, while $\delta(\cdot)$ is the Dirac function. We denote by $I_{0}$ the modified zero-order Bessel function, that is,

$$
I_{0}(z)=\sum_{n=0}^{\infty} \frac{z^{2 n}}{2^{2 n}(n !)^{2}} .
$$


In particular, equality (2.2) means that the particle reaches the points $y= \pm t$ with equal probabilities $e^{-\mu t} / 2$. The absolutely continuous component of distribution (2.2) is concentrated on the interval $(-t, t)$ and is given by

$$
\begin{aligned}
q_{1, a c}(y, t) & \equiv q_{1, a c}^{\mu}(y, t) \\
& =\frac{e^{-\mu t}}{2}\left[\mu I_{0}\left(\mu \sqrt{t^{2}-y^{2}}\right)+\frac{\partial}{\partial t} I_{0}\left(\mu \sqrt{t^{2}-y^{2}}\right)\right] \cdot \chi_{[-t, t]}(y) .
\end{aligned}
$$

In the case of a planar motion, we consider a particle that can move in any of the following four directions:

$$
D_{k}=\left(\cos \frac{k \pi}{2}, \sin \frac{k \pi}{2}\right), \quad k=0,1,2,3 .
$$

We assume that the particle starts at time $t=0$ from the origin and chooses one of the four directions with equal probability $1 / 4$. The changes of directions take place at the Poisson random times $\tau_{i}$ when the particle changes direction $D_{k}$ for $D_{k \pm 1}$ (this means that the particle starts moving along the line being orthogonal to the previous one). In doing so, the particle chooses the directions $D_{k-1}$ and $D_{k+1}$ with equal probabilities $1 / 2$. Between the times $\tau_{i}$, the particle is moving with the constant unit velocity. The current position of the moving particle at time $t$ is denoted by $\mathbf{U}(t)=\left(U_{1}(t), U_{2}(t)\right)$.

In order to obtain the distribution of $\mathbf{U}(t)$ we use the following equivalent representation (see [13]). Let

$$
V_{1}(t)=\xi_{1} \int_{0}^{t}(-1)^{N_{1}(s)} d s, \quad V_{2}(t)=\xi_{2} \int_{0}^{t}(-1)^{N_{2}(s)} d s
$$

be two independent one-dimensional telegraph processes. This means that the random variables $\xi_{i}, i=1,2$, assume values \pm 1 with probabilities $1 / 2$ and that the homogeneous Poisson processes $N_{i}(t), i=1,2$, have rate $\mu / 2$ and are mutually independent. The process

where

$$
\mathbf{U}^{\prime}=\left(U_{1}^{\prime}(t), U_{2}^{\prime}(t)\right)
$$

$$
U_{1}^{\prime}(t)=\frac{V_{1}(t)+V_{2}(t)}{2} \text { and } \quad U_{2}^{\prime}(t)=\frac{V_{1}(t)-V_{2}(t)}{2},
$$

is stochastically equivalent to the process $\mathbf{U}=\mathbf{U}(t), t>0$. This is the case, since the Poisson process $N=N(t)$ (with rate $\mu$ ) governing the changes of directions for $\mathbf{U}=\mathbf{U}(t)$ can be decomposed into two independent Poisson processes $N_{1}$ and $N_{2}$ (each with rate $\mu / 2)$ governing the evolution of the pair $\left(V_{1}, V_{2}\right)$.

Representation (2.5) allows one to obtain the distribution of $\mathbf{U}=\mathbf{U}(t), t>0$, in the following form:

$$
\begin{gathered}
q_{2}(\mathbf{y}, t)=2 q_{1, a c}^{\mu / 2}\left(y_{1}-y_{2}, t\right) q_{1, a c}^{\mu / 2}\left(y_{1}+y_{2}, t\right) \\
+e^{-\mu t / 2}\left[q_{1, a c}^{\mu / 2}\left(y_{1}-y_{2}, t\right)\left\{\delta\left(y_{1}+y_{2}-t\right)+\delta\left(y_{1}+y_{2}+t\right)\right\}\right. \\
\left.+q_{1, a c}^{\mu / 2}\left(y_{1}+y_{2}, t\right)\left\{\delta\left(y_{1}-y_{2}-t\right)+\delta\left(y_{1}-y_{2}+t\right)\right\}\right] \\
+\frac{e^{-\mu t}}{4}\left[\delta\left(y_{1}\right)\left\{\delta\left(y_{2}-t\right)+\delta\left(y_{2}+t\right)\right\}+\delta\left(y_{2}\right)\left\{\delta\left(y_{1}-t\right)+\delta\left(y_{1}+t\right)\right\}\right], \\
\mathbf{y}=\left(y_{1}, y_{2}\right) \in \mathbb{R}^{2}, \quad t>0 .
\end{gathered}
$$

The first term on the right hand side of (2.6) corresponds to the absolutely continuous component of the distribution of the random variable $\mathbf{U}(t)$ assuming values in the square

$$
Q_{t}=\left\{\mathbf{y}=\left(y_{1}, y_{2}\right) \in \mathbb{R}^{2}:\left|y_{1}-y_{2}\right|<t,\left|y_{1}+y_{2}\right|<t\right\} .
$$


The other terms correspond to the discrete and singular components of the distribution concentrated on the boundary $\partial Q_{t}$.

The absolutely continuous component of the distribution is given by

$$
\begin{gathered}
q_{2, a c}(\mathbf{y}, t)=2 q_{1, a c}^{\mu / 2}\left(y_{1}-y_{2}, t\right) q_{1, a c}^{\mu / 2}\left(y_{1}+y_{2}, t\right) \\
=\frac{e^{-\mu t}}{2}\left[\frac{\mu^{2}}{4} I_{0}\left(\frac{\mu}{2} \sqrt{t^{2}-\left(y_{1}-y_{2}\right)^{2}}\right) I_{0}\left(\frac{\mu}{2} \sqrt{t^{2}-\left(y_{1}+y_{2}\right)^{2}}\right)\right. \\
+\frac{\mu}{2} \frac{\partial}{\partial t}\left\{I_{0}\left(\frac{\mu}{2} \sqrt{t^{2}-\left(y_{1}-y_{2}\right)^{2}}\right) I_{0}\left(\frac{\mu}{2} \sqrt{t^{2}-\left(y_{1}+y_{2}\right)^{2}}\right)\right\} \\
\left.+\frac{\partial}{\partial t} I_{0}\left(\frac{\mu}{2} \sqrt{t^{2}-\left(y_{1}-y_{2}\right)^{2}}\right) \frac{\partial}{\partial t} I_{0}\left(\frac{\mu}{2} \sqrt{t^{2}-\left(y_{1}+y_{2}\right)^{2}}\right)\right], \\
\mathbf{y}=\left(y_{1}, y_{2}\right) \in Q_{t},
\end{gathered}
$$

which coincides with the result (3.5) of 13 (for $c=1$ ). The distribution on the boundary $\partial Q_{t}$ can be represented in a similar manner (see (3.7)-(3.8) in [13]).

The stochastic process $U=U(t)$ defined by (2.1) for the one-dimensional case and the process $\mathbf{U}=\mathbf{U}(t)$ described above for the two-dimensional case are called the standard telegraph processes on the line and in the plane, respectively.

The main aim of this paper is to find out how motions in inhomogeneous media (as well as their distributions) can be expressed in terms of the standard telegraph processes. The main tool for deriving these expressions is an underlying rectifying diffeomorphism.

\section{Change of the time. The one-Dimensional CASE}

We start the construction of rectifying diffeomorphisms for the one-dimensional case. It is natural to speak about the random change of time in this case.

Let $c=c(x), x \in(-\infty, \infty)$, be a nonrandom positive continuous function. Let the stochastic process $X=X^{x}(t)$ be defined by the following equation:

$$
X^{x}(t)=x+\xi \int_{0}^{t}(-1)^{N(s)} c\left(X^{x}(s)\right) d s, \quad t \geq 0 .
$$

The process $X=X^{x}(t), t \geq 0$, can alternatively be rewritten as follows:

$$
X^{x}(t)=x+\int_{0}^{t} c\left(X^{x}(s)\right) d U(s) .
$$

Considering the latter representation, one can say that the process $X=X^{x}(t)$ is a motion

of a telegraph particle with space-dependent velocity. The integral equation (3.2) can be rewritten in the equivalent differential form as follows:

$$
\left\{\begin{array}{l}
d X^{x}(t)=c\left(X^{x}(t)\right) d U(t), \quad t>0, \\
X^{x}(0)=x
\end{array}\right.
$$

The distribution of $X=X^{x}(t), t \geq 0$, is evaluated in [18, equality (5.7)]. Below we provide a different proof of this result. Consider the function $\lambda=\lambda^{x}(t)$, which is a solution of the following Cauchy problem:

$$
\left\{\begin{array}{l}
\frac{d \lambda^{x}}{d t}(t)=c\left(\lambda^{x}(t)\right), \quad t>0, \\
\lambda^{x}(0)=x .
\end{array}\right.
$$

Now we change the argument of the function $\lambda=\lambda^{x}(t)$ for a random time. Consider the process

$$
\tilde{X}^{x}(t)=\lambda^{x}(U(t)), \quad t \geq 0 .
$$


Note that the process $\tilde{X}^{x}$ coincides in distribution with $X^{x}$. To prove this we use the relations

$$
d \tilde{X}^{x}(t)=c\left(\lambda^{x}(U(t))\right) d U(t)=c\left(\tilde{X}^{x}(t)\right) d U(t)
$$

which are true almost everywhere with respect to $t$.

Note also that $\lambda=\lambda^{x}(t)$ increases in $t$ (for every fixed $x$ ). Thus the equation $\lambda^{x}(t)=y$ has a unique solution $t=\sigma(y, x)$, whence

$$
\lambda^{x}(\sigma(y, x))=y .
$$

Using the function $\sigma$ one can invert (3.5) and obtain the following equality:

$$
U(t)=\sigma\left(X^{x}(t), x\right) .
$$

It follows from (3.4) that

$$
\sigma(y, x)=\int_{x}^{y} \frac{d z}{c(z)}
$$

Since

$$
\frac{\partial \sigma(y, x)}{\partial y}=\frac{1}{c(y)}
$$

in view of (3.8), we deduce from (3.7) that the transient probabilities of the process $X=X^{x}(t)$ are

$$
p_{1}(y, t \mid x)=\frac{1}{c(y)} q_{1}(\sigma(y, x), t)
$$

Considering the identity

$$
\delta(\sigma(y, x)-t)=c(y) \delta\left(y-\lambda^{x}(t)\right)
$$

we derive the distribution of $X^{x}(t)$ from (2.2):

$$
\begin{aligned}
& p_{1}(y, t \mid x) \\
& =\frac{1}{2} e^{-\mu t}\left[\delta\left(y-\lambda^{x}(t)\right)+\delta\left(y-\lambda^{x}(-t)\right)\right. \\
& +\frac{1}{c(y)}\left(\mu I_{0}\left(\mu \sqrt{t^{2}-\sigma(y, x)^{2}}\right)+\frac{\partial}{\partial t} I_{0}\left(\mu \sqrt{t^{2}-\sigma(y, x)^{2}}\right)\right) \\
& \left.\times \chi_{\left[\lambda^{x}(-t), \lambda^{x}(t)\right]}(y)\right] .
\end{aligned}
$$

Note that the assumptions

$$
\int_{-\infty}^{0} \frac{d z}{c(z)}=+\infty, \quad \int_{0}^{+\infty} \frac{d z}{c(z)}=+\infty
$$

yield that a solution of equation (3.1) exists for all $t>0$. If the above relations do not hold, then a solution of (3.1) exists in a finite interval $\left[0, t_{*}\right)$, where

$$
t_{*}=t_{*}(x)=\min \left(l_{-}, l_{+}\right)
$$

and

$$
l_{-}=\int_{-\infty}^{x} \frac{d z}{c(z)}, \quad l_{+}=\int_{x}^{+\infty} \frac{d z}{c(z)} .
$$

This means that the process $X=X^{x}(t)$ defined by (3.1) approaches infinity in a finite time with probability $1-\mathrm{P}\left\{-t_{*}<U(t)<t_{*}\right\}$. In the physical literature this phenomenon is known as a blowup. 


\section{The TWO-DIMEnSIONAL CASE}

4.1. Two-dimensional telegraph process in an inhomogeneous plane. We use the same approach as in the one-dimensional case. Consider the four continuous functions $c^{i j}=c^{i j}(\mathbf{x}), \mathbf{x}=\left(x_{1}, x_{2}\right) \in \mathbb{R}^{2}, i, j=1,2$, and define a planar motion $\mathbf{X}=\mathbf{X}^{\mathbf{x}}(t), t>0$, by means of the following equations:

$$
\left\{\begin{array}{l}
d X_{1}^{\mathbf{x}}(t)=c^{11}\left(X_{1}^{\mathbf{x}}, X_{2}^{\mathbf{x}}\right) d U_{1}(t)+c^{12}\left(X_{1}^{\mathbf{x}}, X_{2}^{\mathbf{x}}\right) d U_{2}(t), \\
d X_{2}^{\mathbf{x}}(t)=c^{21}\left(X_{1}^{\mathbf{x}}, X_{2}^{\mathbf{x}}\right) d U_{1}(t)+c^{22}\left(X_{1}^{\mathbf{x}}, X_{2}^{\mathbf{x}}\right) d U_{2}(t)
\end{array}\right.
$$

and initial data

$$
\left.\mathbf{X}^{\mathbf{x}}\right|_{t=0}=\left(X_{1}^{\mathbf{x}}(0), X_{2}^{\mathbf{x}}(0)\right)=\mathbf{x}, \quad \mathbf{x}=\left(x_{1}, x_{2}\right) \in \mathbb{R}^{2} .
$$

Here we denote by $U_{1}$ and $U_{2}$ the components of the standard telegraph process $\mathbf{U}$ in the plane.

The system of differential equations (4.1) with initial data (4.2) is equivalent to the following system of integral equations:

$$
\left\{\begin{aligned}
X_{1}^{\mathbf{x}}(t)= & x_{1}+\int_{0}^{t} c^{11}\left(X_{1}^{\mathbf{x}}(s), X_{2}^{\mathbf{x}}(s)\right) d U_{1}(s) \\
& +\int_{0}^{t} c^{12}\left(X_{1}^{\mathbf{x}}(s), X_{2}^{\mathbf{x}}(s)\right) d U_{2}(s) \\
X_{2}^{\mathbf{x}}(t)= & x_{2}+\int_{0}^{t} c^{21}\left(X_{1}^{\mathbf{x}}(s), X_{2}^{\mathbf{x}}(s)\right) d U_{1}(s) \\
& +\int_{0}^{t} c^{22}\left(X_{1}^{\mathbf{x}}(s), X_{2}^{\mathbf{x}}(s)\right) d U_{2}(s)
\end{aligned}\right.
$$

(compare with (3.2)-(3.3)).

This means that the particle starts from the point $\mathbf{x}=\left(x_{1}, x_{2}\right) \in \mathbb{R}^{2}$ and moves according to the velocity fields $\mathbf{c}^{\mathbf{1}}=\left(c^{11}, c^{21}\right)$ and $\mathbf{c}^{\mathbf{2}}=\left(c^{12}, c^{22}\right)$ (backward and forward directions are chosen with equal probabilities); the particle switches from one velocity field to the other after each Poisson event.

The problem is to obtain the distribution of a particle whose movement is described by equations (4.1). The solution of this problem is not as easy as in the one-dimensional case.

4.2. Rectifiable vector fields. We recall some notions and results of the theory of vector fields.

Definition 4.1. Let $\mathbf{c}: \mathbb{R}^{2} \rightarrow \mathbb{R}^{2}$ be a smooth vector field in the plane. We say that the vector field $\mathbf{c}$ is globally rectifiable if there exists a diffeomorphism $\mathcal{F}=\left(F_{1}, F_{2}\right)$ in the whole plane such that $\mathcal{F}_{*} \mathbf{c}=\mathbf{e}$. Here $\mathcal{F}_{*}$ is the differential of the mapping $\mathcal{F}$ and $\mathbf{e}$ is a coordinate unit vector in $\mathbb{R}^{2}$.

Note that any smooth vector field is locally rectifiable in a neighborhood of every nonsingular point (see, for example, [1]).

Definition 4.2. Let $\mathbf{c}_{1}$ and $\mathbf{c}_{2}$ be two smooth vector fields, which are linearly independent in the domain $\Omega \subset \mathbb{R}^{2}$. We say that a pair of vector fields is rectifiable in the domain $\Omega \subset \mathbb{R}^{2}$ if there exists a diffeomorphism $\mathcal{F}=\left(F_{1}, F_{2}\right)$ defined on $\Omega$ and such that $\mathcal{F}_{*} \mathbf{c}_{1}=\mathbf{e}_{1}$ and $\mathcal{F}_{*} \mathbf{c}_{\mathbf{2}}=\mathbf{e}_{\mathbf{2}}$, where $\mathbf{e}_{1}$ and $\mathbf{e}_{\mathbf{2}}$ form a Euclidean basis in the plane $\mathbb{R}^{2}$. If $\Omega=\mathbb{R}^{2}$, then the pair of vector fields is called globally rectifiable.

Rewriting equations (4.1) in the vector form we get

$$
d \mathbf{X}^{\mathbf{x}}(t)=\mathcal{C}\left(\mathbf{X}^{\mathbf{x}}\right) d \mathbf{U}(t),\left.\quad \mathbf{X}^{\mathbf{x}}\right|_{t=0}=\mathbf{x}=\left(x_{1} x_{2}\right) \in \mathbb{R}^{2},
$$

where $\mathbf{X}^{\mathbf{x}}(t)=\left(X_{1}^{\mathbf{x}}(t), X_{2}^{\mathbf{x}}(t)\right)^{T}$ and the matrix $\mathcal{C}(\mathbf{x})$ is given by

$$
\mathcal{C}(\mathbf{x})=\left(\begin{array}{ll}
c^{11}(\mathbf{x}) & c^{12}(\mathbf{x}) \\
c^{21}(\mathbf{x}) & c^{22}(\mathbf{x})
\end{array}\right)
$$


Assume that a pair of vector fields $\mathbf{c}_{\mathbf{1}}$ and $\mathbf{c}_{\mathbf{2}}$ is rectifiable. Let $\mathcal{F}$ be the corresponding diffeomorphism and let $\mathcal{F}_{*}$ be its differential. Definition 4.2 means that $\mathcal{F}_{*} \mathcal{C}=\mathcal{E}$, where the symbol $\mathcal{E}$ stands for the unit matrix. Therefore the tangent mapping $\mathcal{F}_{*}$ is the inverse to $\mathcal{C}$ :

$$
\mathcal{F}_{*}=\left(\begin{array}{ll}
\frac{\partial F_{1}}{\partial x_{1}} & \frac{\partial F_{1}}{\partial x_{2}} \\
\frac{\partial F_{2}}{\partial x_{1}} & \frac{\partial F_{2}}{\partial x_{2}}
\end{array}\right)=\mathcal{C}^{-1}=\left(\begin{array}{ll}
\bar{c}^{11} & \bar{c}^{12} \\
\bar{c}^{21} & \bar{c}^{22}
\end{array}\right) .
$$

Thus the usual Cauchy-Riemann conditions

$$
\frac{\partial \bar{c}^{11}}{\partial x_{2}}=\frac{\partial \bar{c}^{12}}{\partial x_{1}}, \quad \frac{\partial \bar{c}^{21}}{\partial x_{2}}=\frac{\partial \bar{c}^{22}}{\partial x_{1}}
$$

for the elements of the inverse matrix $\mathcal{C}^{-1}$ are necessary and sufficient for the rectifiability of system (4.1).

Let $\mathbf{X}=\mathbf{X}^{\mathbf{x}}(t), t \geq 0$, be a process of the form (4.1) and $Q_{t}(\mathbf{x})$ be the support of the distribution of $\mathbf{X}^{\mathbf{x}}(t)$. Let $\mathbf{c}_{\mathbf{1}}=\left(c^{11}, c^{21}\right)$ and $\mathbf{c}_{\mathbf{2}}=\left(c^{12}, c^{22}\right)$ be a pair of rectifiable vector fields in some domain $\Omega \subset \mathbb{R}^{2}$ (in the sense of Definition 4.2) and let $\mathcal{F}$ be the corresponding diffeomorphism for $\left(\mathbf{c}_{\mathbf{1}}, \mathbf{c}_{\mathbf{2}}\right)$ in the domain $\Omega$.

Theorem 4.1. Let $\Omega \supset Q_{t}(\mathbf{x})$ for $t \leq t_{*}$. Then the distribution density $p_{2}=p_{2}(\mathbf{y}, t \mid \mathbf{x})$ of the stochastic process $\mathbf{X}=\mathbf{X}^{\mathbf{x}}(t)$ is equal to

$$
\begin{gathered}
p_{2}(\mathbf{y}, t \mid \mathbf{x})=\left|\operatorname{det} \mathcal{F}_{*}(\mathbf{y})\right| \cdot q_{2}(\mathcal{F}(\mathbf{y})-\mathcal{F}(\mathbf{x}), t), \\
\mathbf{x}, \mathbf{y} \in Q_{t}(\mathbf{x}) \subset \Omega \subset \mathbb{R}^{2},
\end{gathered}
$$

for $t \leq t_{*}$, where $q_{2}=q_{2}(\mathbf{z}, t), \mathbf{z} \in \mathbb{R}^{2}$, is the distribution density of the standard twodimensional telegraph process defined by (2.6).

Proof. Consider the process $\tilde{\mathbf{X}}=\mathcal{F}(\mathbf{X})$. Applying (4.1) and (4.4) we get

$$
d \tilde{\mathbf{X}}=d \mathcal{F}(\mathbf{X})=\mathcal{F}_{*}(\mathbf{X}) d \mathbf{X}=\mathcal{F}_{*} \mathcal{C} d \mathbf{U}=d \mathbf{U} .
$$

Thus $\tilde{\mathbf{X}}^{\mathbf{x}}(t)=\mathcal{F}(\mathbf{x})+\mathbf{U}(t), t \geq 0$, and the distribution density $\tilde{p}_{2}=\tilde{p}_{2}(\mathbf{y}, t \mid \mathbf{x})$ of the process $\tilde{\mathbf{X}}=\tilde{\mathbf{X}}^{\mathbf{x}}(t)$ is given by

$$
\tilde{p}_{2}(\mathbf{y}, t \mid \mathbf{x})=q_{2}\left(y_{1}-F_{1}(\mathbf{x}), y_{2}-F_{2}(\mathbf{x}), t\right) .
$$

This implies that the distribution density $p_{2}(\mathbf{y}, t \mid \mathbf{x})$ of the random variable $\mathbf{X}^{\mathbf{x}}=\mathcal{F}^{-1} \tilde{\mathbf{X}}^{\mathbf{x}}$ satisfies (4.6).

Remark 4.1. The approach we used for the two-dimensional case coincides with that for the one-dimensional case. The function $\sigma(\cdot, x)$ is the rectifying diffeomorphism for $c(\cdot)$ in the one-dimensional case. Equality (3.9) is a special case of (4.6).

The two-dimensional model is more complicated than the one-dimensional model. The rectifying diffeomorphism does not always exist for a pair of vector fields. Below we consider some examples of rectifiable motions in the plane that satisfy the CauchyRiemann conditions (4.5).

4.3. Examples. 1. Consider a random motion described by the following equations:

$$
\left\{\begin{array}{l}
d X_{1}^{\mathbf{x}}(t)=c^{1}\left(X_{1}^{\mathbf{x}}\right) d U_{1}(t), \\
d X_{2}^{\mathbf{x}}(t)=c^{2}\left(X_{2}^{\mathbf{x}}\right) d U_{2}(t), \quad \mathbf{x}=\left(x_{1}, x_{2}\right) \in \mathbb{R}^{2},
\end{array}\right.
$$

and initial data (4.2). This random motion corresponds to the process defined by (4.1) with two orthogonal fields of velocity $\mathbf{c}_{\boldsymbol{1}}=\left(c^{1}\left(x_{1}\right), 0\right)$ and $\mathbf{c}_{\mathbf{2}}=\left(0, c^{2}\left(x_{2}\right)\right)$ where $c^{1}=c^{1}\left(x_{1}\right)$ and $c^{2}=c^{2}\left(x_{2}\right)$ are some positive continuous functions. 
The model introduced by (4.7) corresponds to the movement of a particle that takes the position

$$
\mathbf{x}=\left(x_{1}, x_{2}\right) \in \mathbb{R}^{2}
$$

at the beginning and may move horizontally with velocity $c^{1}\left(x_{1}\right)$ and vertically with velocity $c^{2}\left(x_{2}\right)$ (in both directions). Note that the horizontal velocity depends on the first coordinate $x_{1}$ only, while the vertical velocity depends on $x_{2}$ only (the case of $c^{1}, c^{2}=$ const is studied in detail in [14]). The model defined by (4.7) corresponds to a movement in an anisotropic medium.

It is clear that the process $X_{1}=X_{1}^{\mathbf{x}}(t) \equiv X_{1}^{x_{1}}(t)$ does not depend on $x_{2}$, while the process

$$
X_{2}=X_{2}^{\mathbf{x}}(t) \equiv X_{2}^{x_{2}}(t)
$$

does not depend on $x_{1}$. Therefore equation (4.7) can be rewritten in the following equivalent form:

$$
\left\{\begin{array}{l}
X_{1}^{x_{1}}(t)=x_{1}+\int_{0}^{t} c^{1}\left(X_{1}^{x_{1}}(s)\right) d U_{1}(s), \\
X_{2}^{x_{2}}(t)=x_{2}+\int_{0}^{t} c^{2}\left(X_{2}^{x_{2}}(s)\right) d U_{2}(s) .
\end{array}\right.
$$

We use Theorem 4.1 to obtain the distribution densities $p_{2}=p_{2}(\mathbf{y}, t \mid \mathbf{x}), \mathbf{x}, \mathbf{y} \in \mathbb{R}^{2}$, for this process. The matrix $\mathcal{C}$ becomes of the form

$$
\mathcal{C}=\left(\begin{array}{cc}
c^{1}\left(x_{1}\right) & 0 \\
0 & c^{2}\left(x_{2}\right)
\end{array}\right)
$$

and hence

$$
\mathcal{F}_{*}=\mathcal{C}^{-1}=\left(\begin{array}{cc}
c^{1}\left(x_{1}\right)^{-1} & 0 \\
0 & c^{2}\left(x_{2}\right)^{-1}
\end{array}\right) .
$$

It is easy to see that the rectifying mapping $\mathcal{F}=\left(F_{1}, F_{2}\right)$ can be rewritten for this case as follows:

$$
F_{1}(\mathbf{y}) \equiv F_{1}\left(y_{1}\right)=\int_{0}^{y_{1}} \frac{d z}{c^{1}(z)}, \quad F_{2}(\mathbf{y}) \equiv F_{2}\left(y_{2}\right)=\int_{0}^{y_{2}} \frac{d z}{c^{2}(z)} .
$$

According to the notation introduced in Section 3 we get

$$
\begin{aligned}
& F_{1}\left(y_{1}\right)-F_{1}\left(x_{1}\right) \equiv \sigma_{1}\left(y_{1}, x_{1}\right)=\int_{x_{1}}^{y_{1}} \frac{d z}{c^{1}(z)}, \\
& F_{2}\left(y_{2}\right)-F_{2}\left(x_{2}\right) \equiv \sigma_{2}\left(y_{2}, x_{2}\right)=\int_{x_{2}}^{y_{2}} \frac{d z}{c^{2}(z)} .
\end{aligned}
$$

Thus the distribution of the position of a particle is given by

$$
\begin{aligned}
p_{2}(\mathbf{y}, t \mid \mathbf{x}) & =\frac{1}{c^{1}\left(y_{1}\right) c^{2}\left(y_{2}\right)} q_{2}\left(F_{1}\left(y_{1}\right)-F_{1}\left(x_{1}\right), F_{2}\left(y_{2}\right)-F_{2}\left(x_{2}\right), t\right) \\
& \equiv \frac{1}{c^{1}\left(y_{1}\right) c^{2}\left(y_{2}\right)} q_{2}\left(\sigma_{1}\left(y_{1}, x_{1}\right), \sigma_{2}\left(y_{2}, x_{2}\right), t\right),
\end{aligned}
$$

where the function $q_{2}=q_{2}\left(\sigma_{1}, \sigma_{2}, t\right)$ is defined by (2.6) . 
In particular, the absolutely continuous component of the distribution is

$$
\begin{aligned}
& p_{2, a c}(\mathbf{y}, t \mid \mathbf{x})= \frac{e^{-\mu t}}{2 c^{1}\left(y_{1}\right) c^{2}\left(y_{2}\right)} \\
& \times\left[\frac{\mu^{2}}{4} I_{0}\left(\frac{\mu}{2} \sqrt{t^{2}-\left(\sigma_{1}-\sigma_{2}\right)^{2}}\right) I_{0}\left(\frac{\mu}{2} \sqrt{t^{2}-\left(\sigma_{1}+\sigma_{2}\right)^{2}}\right)\right. \\
& \quad+\frac{\mu}{2} \frac{\partial}{\partial t}\left\{I_{0}\left(\frac{\mu}{2} \sqrt{t^{2}-\left(\sigma_{1}-\sigma_{2}\right)^{2}}\right) I_{0}\left(\frac{\mu}{2} \sqrt{t^{2}-\left(\sigma_{1}+\sigma_{2}\right)^{2}}\right)\right\} \\
&\left.\quad+\frac{\partial}{\partial t} I_{0}\left(\frac{\mu}{2} \sqrt{t^{2}-\left(\sigma_{1}-\sigma_{2}\right)^{2}}\right) \frac{\partial}{\partial t} I_{0}\left(\frac{\mu}{2} \sqrt{t^{2}-\left(\sigma_{1}+\sigma_{2}\right)^{2}}\right)\right]
\end{aligned}
$$

for $\left|\sigma_{1}-\sigma_{2}\right|<t$ and $\left|\sigma_{1}+\sigma_{2}\right|<t$, where $\sigma_{1}=\sigma_{1}\left(y_{1}, x_{1}\right)$ and $\sigma_{2}=\sigma_{2}\left(y_{2}, x_{2}\right)$. The singular component of the distribution $p_{2}=p_{2}(\mathbf{y}, t \mid \mathbf{x})$ can also be easily obtained.

Remark 4.2. One can look at these formulas from another point of view similar to that discussed in Section 2. Namely, consider the solutions $\lambda_{1}=\lambda_{1}^{x_{1}}(t)$ and $\lambda_{2}=\lambda_{2}^{x_{2}}(t)$ of the following Cauchy problems:

$$
\begin{cases}\frac{d \lambda_{1}^{x_{1}}}{d t}(t)=c^{1}\left(\lambda_{1}^{x_{1}}(t)\right), & t>0, \\ \lambda_{1}^{x_{1}}(0)=x_{1}, & x_{1} \in(-\infty, \infty),\end{cases}
$$

and

$$
\begin{cases}\frac{d \lambda_{2}^{x_{2}}}{d t}(t)=c^{2}\left(\lambda_{2}^{x_{2}}(t)\right), & t>0, \\ \lambda_{2}^{x_{2}}(0)=x_{2}, & x_{2} \in(-\infty, \infty) .\end{cases}
$$

Let $\sigma_{1}=\sigma_{1}\left(y_{1}, x_{1}\right)$ and $\sigma_{2}=\sigma_{2}\left(y_{2}, x_{2}\right)$ be the inverse functions to $\lambda_{1}$ and $\lambda_{2}$, respectively:

$$
\lambda_{1}^{x_{1}}\left(\sigma_{1}\left(y_{1}, x_{1}\right)\right)=y_{1}, \quad \lambda_{2}^{x_{2}}\left(\sigma_{2}\left(y_{2}, x_{2}\right)\right)=y_{2} .
$$

It is clear that these functions coincide with $\sigma_{1}$ and $\sigma_{2}$ defined in (4.12)-(4.13).

The domain $Q_{t}=\left\{\mathbf{y} \in \mathbb{R}^{2}:\left|\sigma_{1}-\sigma_{2}\right|<t,\left|\sigma_{1}+\sigma_{2}\right|<t\right\}$ consisting of points $\mathbf{y}$ attainable by the moving particle in time $t$ is a set whose boundary has, in general, a rather irregular form.

2. Now we consider a slightly different model. Let a planar motion be defined by the following equations:

$$
\left\{\begin{array}{l}
d X_{1}^{\mathbf{x}}(t)=c^{1}\left(X_{1}^{\mathbf{x}}\right)\left(d U_{1}(t)+d U_{2}(t)\right), \\
d X_{2}^{\mathbf{x}}(t)=c^{2}\left(X_{2}^{\mathbf{x}}\right)\left(d U_{1}(t)-d U_{2}(t)\right)
\end{array}\right.
$$

with initial data (4.2). It is easy to see that this process satisfies the assumptions of Theorem 4.1 but the solution of system (4.19) can be constructed in a simpler way.

As mentioned above (see (2.4)-(2.5)), the process defined by equations (4.19) is stochastically equivalent to the process described by the equations

$$
\left\{\begin{array}{l}
d X_{1}^{\mathbf{x}}(t)=c^{1}\left(X_{1}^{\mathbf{x}}\right) d V_{1}(t), \\
d X_{2}^{\mathbf{x}}(t)=c^{2}\left(X_{2}^{\mathbf{x}}\right) d V_{2}(t),
\end{array}\right.
$$

where $V_{1}=V_{1}(t)$ and $V_{2}=V_{2}(t)$ are two independent standard telegraph processes in the line with rate $\mu / 2$ instead of $\mu$. Hence the process (4.19) splits into two independent one-dimensional motions $X_{2}=X_{2}^{x_{2}}(t)$ with space-varying velocities $c_{1}\left(x_{1}\right)$ and $c_{2}\left(x_{2}\right)$, 
respectively. This allows us to obtain the distribution explicitly:

$$
\begin{aligned}
p_{2}(\mathbf{y}, t \mid \mathbf{x}) & =\frac{1}{2 c^{1}\left(y_{1}\right) c^{2}\left(y_{2}\right)} q_{2}\left(z_{1}, z_{2}, t\right) \\
& =\frac{1}{c^{1}\left(y_{1}\right) c^{2}\left(y_{2}\right)} q_{1}^{\mu / 2}\left(\sigma_{1}\left(y_{1}, x_{1}\right), t\right) \cdot q_{1}^{\mu / 2}\left(\sigma_{2}\left(y_{2}, x_{2}\right), t\right),
\end{aligned}
$$

where

$$
z_{1}=\frac{\sigma_{1}\left(y_{1}, x_{1}\right)+\sigma_{2}\left(y_{2}, x_{2}\right)}{2}, \quad z_{2}=\frac{\sigma_{1}\left(y_{1}, x_{1}\right)-\sigma_{2}\left(y_{2}, x_{2}\right)}{2} .
$$

Here $q_{2}=q_{2}\left(z_{1}, z_{2}, t\right)$ denotes the distribution density of the standard telegraph process in the plane $\mathbb{R}^{2}$ (see (2.6) $), q_{1}^{\mu / 2}$ is the distribution density of the standard telegraph process in the plane (with rate $\mu / 2$ ), and $\sigma_{1}$ and $\sigma_{2}$ are defined in (4.12)-(4.13) (or in (4.18) $)$.

The rectifying diffeomorphism of vector fields related to this process can be written as follows:

$$
F_{1}(\mathbf{y})=\frac{\sigma_{1}\left(y_{1}, 0\right)+\sigma_{2}\left(y_{2}, 0\right)}{2}, \quad F_{2}(\mathbf{y})=\frac{\sigma_{1}\left(y_{1}, 0\right)-\sigma_{2}\left(y_{2}, 0\right)}{2} .
$$

Applying this diffeomorphism to formula (4.6) we obtain (4.20).

3. Consider another example of a planar motion. Assume that the particle either rotates (both directions, clockwise and counterclockwise, are possible) with angular velocity depending on the current angular position or it moves radially (both directions, outwards and onwards, are possible) with the velocity depending on the current distance from the origin. The particle switches the radial motion to the circular motion (or vice versa) at Poisson times (it chooses one of two possible directions at the switching time with probability $1 / 2)$.

The random motion described above can be represented as follows:

$$
\left\{\begin{array}{l}
d X_{1}^{\mathbf{x}}(t)=-\omega(\theta) X_{2}^{\mathbf{x}} d U_{1}(t)+\frac{c(\rho)}{\rho} X_{1}^{\mathbf{x}} d U_{2}(t), \\
d X_{2}^{\mathbf{x}}(t)=\omega(\theta) X_{1}^{\mathbf{x}} d U_{1}(t)+\frac{c(\rho)}{\rho} X_{2}^{\mathbf{x}} d U_{2}(t)
\end{array}\right.
$$

with initial position $\mathbf{X}^{\mathbf{x}}(0)=\mathbf{x} \neq \mathbf{0}$. Here $\rho=\rho(\mathbf{X})$ and $\theta=\theta(\mathbf{X})$ are polar coordinates of the moving particle $\mathbf{X}=\left(X_{1}^{\mathbf{x}}(t), X_{2}^{\mathbf{x}}(t)\right) ; c=c(\rho)>0$ and $\omega=\omega(\theta)$ denote the radial and angular fields of velocity. To find the corresponding rectifying diffeomorphism one needs to invert the matrix

$$
\mathcal{C}=\left(\begin{array}{cc}
-\omega(\varphi) x_{2} & \frac{c(r)}{r} x_{1} \\
\omega(\varphi) x_{1} & \frac{c(r)}{r} x_{2}
\end{array}\right),
$$

where $r=r(\mathbf{x})$ and $\varphi=\varphi(\mathbf{x})$ are polar coordinates of the point $\mathbf{x}=\left(x_{1}, x_{2}\right) \neq(0,0)$. The inverse matrix is given by

$$
\mathcal{F}_{*}=\mathcal{C}^{-1}=\left(\begin{array}{cc}
\frac{-x_{2}}{\omega(\varphi) r^{2}} & \frac{x_{1}}{\omega(\varphi) r^{2}} \\
\frac{x_{1}}{r c(r)} & \frac{x_{2}}{r c(r)}
\end{array}\right) .
$$

Thus the rectifying diffeomorphism exists in every domain that does not contain the origin. The diffeomorphism $\mathcal{F}=\left(F_{1}, F_{2}\right)$ coincides with the quasi-polar change of variables. More precisely,

$$
F_{1}=F_{1}(\mathbf{x})=\int_{0}^{\varphi(\mathbf{x})} \frac{d \varphi}{\omega(\varphi)}, \quad F_{2}=F_{2}(\mathbf{x})=\int_{0}^{r(\mathbf{x})} \frac{d r}{c(r)}
$$


Theorem 4.1 allows us to obtain the distribution of the position of the particle at least for sufficiently small $t$. Assume that

$$
t<\min \left(\int_{0}^{\pi} \frac{d \varphi}{\omega(\varphi)}, \int_{0}^{r(\mathbf{x})} \frac{d r}{c(r)}\right) .
$$

Then

$$
p_{2}(\mathbf{y}, t \mid \mathbf{x})=\frac{1}{|c(\rho(\mathbf{y})) \omega(\theta(\mathbf{y})) \rho(\mathbf{y})|} q_{2}\left(F_{1}(\mathbf{y})-F_{1}(\mathbf{x}), F_{2}(\mathbf{y})-F_{2}(\mathbf{x}), t\right),
$$

where $q_{2}(\cdot, \cdot, t)$ is the distribution density of the standard motion in the plane and $F_{1}$ and $F_{2}$ are defined in (4.22).

The boundary of the set $Q_{t}$ of points attainable in time $t$ is curvilinear. In particular, if $c(r) \equiv 1$ and $\omega(\varphi) \equiv 1$, then the boundary is formed by the following segments of Archimedes' spirals:

$$
\rho=\rho_{0}+t-\left|\theta-\theta_{0}\right|, \quad \rho=\rho_{0}-t+\left|\theta-\theta_{0}\right| \quad\left|\theta-\theta_{0}\right|<t .
$$

\section{The Underlying PARTial DifFerential EQUations}

The partial differential equations governing the distributions of the standard telegraph processes are well known. For one-dimensional motions, the density $q_{1}=q_{1}(y, t)$ satisfies the telegraph equation

$$
\left\{\begin{array}{l}
\frac{\partial^{2} q_{1}}{\partial t^{2}}+2 \mu \frac{\partial q_{1}}{\partial t}=\frac{\partial^{2} q_{1}}{\partial y^{2}}, \quad t>0 \\
\left.q_{1}\right|_{t=0}=\delta(y),\left.\quad \frac{\partial q_{1}}{\partial t}\right|_{t=0}=0
\end{array}\right.
$$

(see, for example, [6]).

In the planar case, the transition probabilities $q_{2}=q_{2}(\mathbf{y}, t)$ form a solution of a fourthorder hyperbolic equation (see [13]). After the transformation $\bar{q}_{2}=e^{\mu t} q_{2}$, this equation can be written as follows:

$$
\frac{\partial^{4} \bar{q}_{2}}{\partial t^{4}}=\left(\mathcal{L}_{y_{1}}^{2}+\mathcal{L}_{y_{2}}^{2}\right) \frac{\partial^{2} \bar{q}_{2}}{\partial t^{2}}+\mu^{2} \frac{\partial^{2} \bar{q}_{2}}{\partial t^{2}}-\mathcal{L}_{y_{2}}^{2} \mathcal{L}_{y_{1}}^{2} \bar{q}_{2},
$$

where $\mathcal{L}_{y_{i}}^{2}=\partial^{2} / \partial y_{i}{ }^{2}, i=1,2$.

The distributions of the telegraph processes (2.2) and (2.7) can be derived from these two equations.

Note that equation (5.1) follows from (1.1) for $n=1$ if $V=\{-1,+1\}$ and the turning kernel is symmetric, that is, $T(-1,1)=T(1,-1)=1 / 2$. Equation (5.2) follows from (1.1) for $n=2$ if $V=\left\{D_{k}, k=0,1,2,3\right\}$ and $T\left(D_{k}, D_{k-1}\right)=T\left(D_{k}, D_{k+1}\right)=1 / 2$ (the integral on the right hand side of (1.1) turns to the sum for these two particular cases).

Below we derive equations related to random motions in inhomogeneous media. In the one-dimensional case defined by (3.1), the equation follows from the representations (3.5) and (3.9). It is easy to understand that the density

$$
p_{1}=p_{1}(y, t \mid x)
$$

satisfies the Cauchy problem

$$
\left\{\begin{array}{l}
\frac{\partial^{2} p_{1}}{\partial t^{2}}+2 \mu \frac{\partial p_{1}}{\partial t}=\frac{\partial}{\partial y} c(y) \frac{\partial c(y) p_{1}(y, t \mid x)}{\partial y}, \quad t>0, \\
\left.p_{1}\right|_{t=0}=\delta(x-y),\left.\quad \frac{\partial p_{1}}{\partial t}\right|_{t=0}=0 .
\end{array}\right.
$$

Moreover this density is a solution of the equation

$$
\frac{\partial^{2} p_{1}}{\partial t^{2}}+2 \mu \frac{\partial p_{1}}{\partial t}=c(x) \frac{\partial}{\partial x} c(x) \frac{\partial p_{1}(y, t \mid x)}{\partial x}, \quad t>0,
$$


with the same initial data as in (5.3). Note that (5.3) and (5.4) can be rewritten in the form of backward and forward Fokker-Planck equations. Explicit expressions for the solutions of these equations with boundary conditions related to reflection and/or absorption barriers are also known (see [4] or 18] for further details).

In particular, equations (5.3) and (5.4) can be applied to describe a one-dimensional diffusion in a cellular media. Let the velocity of a particle be a function $c=c(x)$, $x \in(-\infty, \infty)$, with period 1. Then the density is given by (3.9)-(3.10). It is interesting to note that, for a spatially scaled function $c^{\varepsilon}(x)=c(x / \varepsilon)$, the corresponding densities have the following asymptotic behavior:

$$
p_{1}^{\varepsilon}(y, t \mid x) \sim \frac{1}{c(y / \varepsilon)} q_{1}((y-x) \sigma, t)
$$

as $\varepsilon \rightarrow 0$, where

$$
\sigma=\int_{0}^{1} \frac{d z}{c(z)}
$$

In the two-dimensional case, the corresponding equation looks much more sophisticated. Let $\mathbf{X}=\left(X_{1}, X_{2}\right)$ be a random motion defined in (4.1) and let $\mathcal{F}$ be the rectifying diffeomorphism for the vector fields $\mathbf{c}_{1}=\left(c^{11}, c^{21}\right)$ and $\mathbf{c}_{2}=\left(c^{12}, c^{22}\right)$ in the domain $\Omega \subset \mathbb{R}^{2}$. The governing equation follows from (5.2) after a suitable change of variables. We have

$$
p_{2}(\mathbf{y}, t \mid \mathbf{x})=\frac{1}{|\operatorname{det} \mathcal{C}(\mathbf{y})|} q_{2}(\mathcal{F}(\mathbf{y})-\mathcal{F}(\mathbf{x}), t), \quad \mathbf{x}, \mathbf{y} \in \Omega,
$$

in view of (4.6).

Since $\mathcal{C}=\left(\mathcal{F}_{*}\right)^{-1}$, we also have

$$
\left.\frac{\partial q_{2}(\mathbf{z}, t)}{\partial z_{i}}\right|_{\mathbf{z}=\mathcal{F}(\mathbf{y})-\mathcal{F}(\mathbf{x})}=\mathcal{L}_{i}\left[q_{2}(\mathcal{F}(\mathbf{y})-\mathcal{F}(\mathbf{x}), t)\right], \quad i=1,2,
$$

where

$$
\mathcal{L}_{1}=c^{11}(\mathbf{y}) \frac{\partial}{\partial y_{1}}+c^{21}(\mathbf{y}) \frac{\partial}{\partial y_{2}}, \quad \mathcal{L}_{2}=c^{12}(\mathbf{y}) \frac{\partial}{\partial y_{1}}+c^{22}(\mathbf{y}) \frac{\partial}{\partial y_{2}} .
$$

Using equalities (5.6) and (5.7) we change the variables in equation (5.2). Put

$$
u(\mathbf{y}, t)=|\operatorname{det} \mathcal{C}(y)| p_{2}(\mathbf{y}, t \mid \mathbf{x}) e^{\mu t} .
$$

Then the function $u$ satisfies the following fourth-order equation:

$$
\frac{\partial^{4} u}{\partial t^{4}}-\mu^{2} \frac{\partial^{2} u}{\partial t^{2}}=\left(\mathcal{L}_{1}^{2}+\mathcal{L}_{2}^{2}\right) \frac{\partial^{2} u}{\partial t^{2}}-\mathcal{L}_{2}^{2} \mathcal{L}_{1}^{2} u
$$

If $\omega=1$ and $c=1$ in Example 3 of the preceding section, then $\mathcal{L}_{1}=\partial / \partial \theta$ and $\mathcal{L}_{2}=\partial / \partial \rho$.

\section{BIBLIOGRAPHY}

1. V. I. Arnol'd, Ordinary Differential Equations, "Nauka", Moscow, 1971; English transl., MIT Press, Cambridge, MA-London, 1978. MR0508209 (58:22707)

2. D. G. Aronson, N. V. Mantzaris, and H. G. Othmer, Wave propagation and blocking in inhomogeneous media, Discrete and Continuous Dynamical Systems 13 (2005), 843-876. MR2166708 (2006k:35287)

3. S. Goldstein, On diffusion by discontinuous movements and the telegraph equation, Quart. J. Mech. Appl. Math. 4 (1951), 129-156. MR0047963(13:960b)

4. K. P. Hadeler, Reaction transport systems in biological modelling, Mathematics Inspired by Biology, 95-150, CIME Lectures 1997, Florence (V. Capasso and O. Diekmann, eds.), Lecture Notes in Mathematics 1714, Springer-Verlag, 1999. MR1737306

5. D. D. Joseph and L. Preziosi, Addendum to the paper: "Heat waves" [Rev. Mod. Phys. 61 (1989), no. 1, 41-73]; Rev. Mod. Phys. 62 (1990), no. 2, 375-391. MR.1056235(91e:80003) 
6. M. Kac, Probability and Related Topics in Physical Sciences, Interscience, London, 1959. MR0102849 (21:1635)

7. M. Kac, A stochastic model related to the telegrapher's equation, Rocky Mountain J. Math. 4 (1974), 497-509. MR0510166 (58:23185)

8. A. Kolesnik and E. Orsingher, Analysis of a finite-velocity planar random motion with reflection, Theory of Probability and its Applications 46 (2001), no. 1, 138-147. MR1968710 (2004g:60109)

9. H. G. Othmer, S. R. Dunbar, and W. Alt, Models of dispersal in biological systems, J. Math. Biol. 26 (1988), 263-298. MR949094 (90a:92064)

10. T. Hillen and H. G. Othmer, The diffusion limit of transport equations derived from velocityjump processes, SIAM J. Appl. Math. 61 (2000), no. 3, 751-775. MR1788017 (2001m:35302)

11. H. G. Othmer and T. Hillen, The diffusion limit of transport equations. II. Chemotaxis equations, SIAM J. Appl. Math. 62 (2002), no. 4, 1222-1250. MR1898520 (2003c:35154)

12. E. Orsingher, Probability law, flow function, maximum distribution of wave-governed random motions and their connections with Kirchoff's law, Stoch. Processes Appl. 34 (1990), 49-66. MR.1039562 (91g:60086)

13. E. Orsingher, Exact joint distribution in a model of planar random motion, Stochastics and Stochastics Reports 69 (2000), no. 1-2, 1-10. MR1751715 (2000m:60117)

14. E. Orsingher and N. Ratanov, Planar random motions with drift, J. Appl. Math. Stoch. Anal. 15 (2002), no. 3, 205-221. MR1930947 (2003i:60113)

15. E. Orsingher, Bessel functions of third order and the distribution of cyclic planar motions with three directions, Stochastics and Stochastics Reports 74 (2002), no. 3-4, 617-631. MR.1943582 (2003j:60144)

16. H. G. Othmer, On significance of finite propagation speeds in multicomponent reacting systems, J. Chem. Phys. 64 (1976), 460-470. MR0449372 (56:7676)

17. M. A. Pinsky, Lectures on Random Evolution, World Scientific, Singapore-New Jersey-London-Hong Kong, 1992. MR.1143780 (93b:60160)

18. N. E. Ratanov, Random walks of a particle in a one-dimensional inhomogeneous environment with reflection and absorption, Teor. Matem. Fiz. 112 (1997), no. 1, 81-91. (Russian) MR:1478901 (98k:82079)

19. N. Ratanov, Reaction-advection random motions in inhomogeneous media, Physica D 189 (2004), 130-140. MR2044720 (2004m:35167)

20. N. Ratanov, Branching random motions, nonlinear hyperbolic systems and travelling waves, European Series in Applied and Industrial Mathematics (ESAIM:PS) 10 (2006), 236-257. MR2219342 (2007g:60101)

21. D. W. Stroock, Some stochastic processes which arise from a model of the motion of a bacterium, Z. Wahrscheinlichkeitstheorie und Verw. Gebiete 28 (1973/74), 303-315. MR0386038 (52:6897)

22. G. H. Weiss, Some applications of persistent random walks and the telegrapher's equation, Physica A 311 (2002), 381-410. MR1943373 (2004h:82096)

Dipartimento di Statistica, Probabilitá e Statistiche Applicate, Universitá degli Studi di Roma "La Sapienza", 00185 Rome, Italy

E-mail address: enzo.orsingher@uniroma1.it

Universidad del Rosario, Bogotá, Colombia

E-mail address: nratanov@urosario.edu.co

Received 16/MAY/2006

Translated by THE AUTHORS 